\title{
LA ABSORCIÓN FUTURA DE FUERZA DE TRABAJO EN MÉXICO*
}

\author{
GERARD K. BoON ** \\ El Colegio de México
}

\section{INTRODUCCIÓN}

El problema económico y social más serio y desafiante al cual se enfrentarán los países en vías de desarrollo dentro de los próximos decenios, será el del empleo. En sentido económico, un desarrollo reducido se manifiesta tanto en la situación del bienestar como en la del empleo en estos países. No se discutirá aquí acerca de todos los bien conocidos hechos y argumentos sobre esta doble característica de la mayoría de los países en desarrollo, sino que más bien la atención será enfocada sobre México en particular.

No hay duda de que la cuestión del empleo es uno de los problemas económicos más graves que afronta este país, si no es que el más grave. El crecimiento económico observado en el período 1940-1970 ha sido satisfactorio, pero el desempleo, abierto y disfrazado, se ha incrementado y la distribución del ingreso se ha hecho más desigual. Las crecientes discrepancias internas en el bienestar de diferentes grupos dentro del mismo país son intolerables, y pueden llevar a serios conflictos sociales en los decenios por venir si no se logra un cambio en los esquemas existentes. El problema de proveer de empleo e ingresos decorosos a todos los mexicanos se agrava por el hecho de que la población crece a una de las tasas más altas del mundo. De hecho no existe otro país con un tamaño de población como el de México -alrededor de 55 millones- que tenga una tasa de crecimiento de población tan alta $-3.5 \%$ anual. De acuerdo con distintas hipótesis, la población de México en el año 2000 llegará a alcanzar alguna de las siguientes cifras proyectadas: 122,140 o 152 millones.

Un crecimiento poblacional de tal magnitud ejerce sobre la economía una gran presión, y en este trabajo se presentan algunos análisis acerca

* Este artículo constituye el capítulo II del libro: La decisión sobre sectores y tecnología en el desarrollo económico, Gerard K. Boon, El Colegio de México, 1974, mimeografiado. Traducción del inglés de Roberto Ham Chande.

** Acerca de este tema he tenido intercambios de opiniones muy ilustrativos con J. Tinbergen, J. Sandee, y A. ten Kate. Sin embargo, todas las omisiones son responsabilidad exclusivamente mía. 
de la cuantificación de la posible situación de oferta y demanda de la población económicamente activa en el próximo decenio.

En la segunda sección de este artículo el análisis abarca el periodo 1970-1982. A través de un modelo matemático, la demanda de mano de obra se analiza para toda la economía, dividida en 15 sectores. En un análisis de este tipo conviene investigar también el balance entre la oferta y la demanda de capital, así como el del comercio exterior y ciertos factores de producción de naturaleza crítica que pudieran implicar restricciones sobre los posibles objetivos en la política del empleo. Estos análisis complementarios no se presentan aquí para no ampliar demasiado el objetivo de la investigación. Sin embargo, los análisis empíricos sobre el empleo que se dan a continuación, suponen una tasa global de crecimiento del PIB en vista de que otras investigaciones han demostrado que el saldo entre inversión y ahorro y la balanza de pagos no son restricciones graves.

Del mismo modo, sería útil investigar el saldo entre la oferta y la demanda de mano de obra desglosada en categorías de calificación o profesión. Tal refinamiento puede introducirse con bastante facilidad aunque no se presenta en este trabajo. ${ }^{1}$ En este capítulo sólo se abordará el problema del empleo en sus relaciones básicas, tratando de contestar la pregunta de si la economía mexicana será capaz de ofrecer trabajo decoroso para sus ciudadanos en los próximos decenios. Si no es muy factible que las tendencias fundamentales lleven a una buena situación en el empleo, se discutirá y analizará entonces qué opciones tendrían probabilidad de éxito para alcanzar el objetivo de empleo e ingreso adecuado para todos.

Es obvio que un buen nivel de empleo puede alcanzarse de varias maneras, como por ejemplo: si se incrementa la tasa global de crecimiento económico; o se cambian la producción para lograr una mayor absorción de mano de obra, o la tecnología de la producción para hacer más intensivo el uso de mano de obra; y por último, aunque no por ello menos importante, frenar la tasa de crecimiento de la población. Es claro que esta última opción es útil sólo a largo plazo, aunque ésta también es válida en el análisis tal y como se verá al estudiarse la situación del empleo en el año 2000.

En la última sección de este artículo se estudiará, en forma muy aproximada, la situación del empleo en el año 2000. En ese año, la población mexicana sobrepasará fácilmente los 100 millones de habitantes y, será de un tercio a un medio del tamaño de la población de Estados Unidos. No hay duda que para los estándares de Europa Occidental o del Sudeste Asiático, México no está sobrepoblado si se considera la medida del número de gentes por kilómetro cuadrado. Sin embargo, debe recordarse que más de una tercera parte del país es inadecuada para ser habitable, y que además se visualiza el problema no desde el punto de vista espacial sino desde el económico. Es claro que la capaci-

1 Véase, por ejemplo, Ref. [6]. 
dad de absorción de mano de obra del país la determina la disponibilidad de factores complementarios de producción en relación con la población en busca de empleo, y no la disponibilidad de espacio.

\section{LA CAPACIDAD DE ABSORCIÓN DE MANO DE OBRA EN EL AÑo 1982}

La población económicamente activa (PEA) de México en 1976 se proyecta a 16820000 gentes al utilizarse una tasa de crecimiento anual de $3.8 \%$, la cual es bastante real. De mantenerse la misma tasa de crecimiento hasta enero de 1982, la PEA sería de $20268000 .^{2}$ Dado el hecho de que la PEA era de 12955000 en enero de 1970, de acuerdo con el Censo General de Población de 1970, para 1982 la economía tendrá que proporcionar empleo para aproximadamente 7.3 millones de gentes más que en 1970. Por desgracia, esta cifra no indica la necesidad total ya que no toma en cuenta el desempleo y el subempleo existente en 1970. Asimismo, el nivel de empleo basado en la producción existente en 1970 disminuirá con el tiempo. Aunque el Censo General de Población de 1970 señala algún desempleo, de 485000 gentes, la realidad es que el desempleo y el subempleo en México es bastante mayor. El hecho es que los datos sobre ocupación de este censo incluyen subempleo, y al momento de relacionar los datos censales de ocupación por sector con la producción bruta o el valor agregado bruto por sector, los coeficientes ocupacionales aparecen relativamente altos pues incluyen la fuerza de trabajo subempleada. Este problema no presenta la misma gravedad para cada sector, ya que los datos estarán en general más correctos en los sectores de transformación o industriales, que en los sectores primarios o terciarios. De cualquier modo, en un análisis del empleo es necesario estimar el desempleo inicial y tomar en consideración los cambios en el tiempo de la productividad de la mano de obra, la cual pudiera también incluir el aspecto del subempleo dependiendo del significado de los datos. Así, además del incremento usual de la productividad de la mano de obra en el tiempo, debe considerarse también el desempleo y el subempleo de la PEA existente en el año base de análisis.

Es cierto que en México, como en cualquier otro país, los datos sobre el empleo constituyen uno de los obstáculos más serios que se oponen a la obtención de estimaciones confiables acerca del nivel del empleo y de la capacidad de la economía para absorber la mano de obra en el tiempo. Una manera de subsanar esta deficiencia es mediante el uso de coeficientes de mano de obra que se derivan y proyectan con base en las funciones de producción CES, efectuadas en forma transversal entre países, o utilizando datos sobre tecnología en la economía. En el análisis que se presenta en este trabajo, se utilizan las productividades del trabajo, proyectadas mediante el CES y otras funciones estadísticas, de acuerdo con los procedimientos presentados en [5], para los sectores de minería

2 En este análisis todas las cifras se han redondeado. 
y extracción, construcción, y los siete sectores de transformación. Además, se utiliza otro conjunto de datos proyectados.

\section{Modelos de absorción de mano de obra para 1982}

Existen varios procedimientos posibles para estimar la oferta y la demanda de fuerza de trabajo para 1982, de los cuales se discutirán dos versiones. En uno se trabaja con incrementos en el período 1970-1982; otro estima en forma directa la demanda para 1982. El análisis por incremento es conceptualmente más difícil y requiere de mayor información. Por otra parte, este análisis brinda mayor información ya que la oferta de trabajo se desglosa en tres componentes:

a) el desempleo en el año base;

b) i) la fuerza de trabajo sustraída por el incremento en la productividad en el período de análisis; $i i)$ el desempleo en el período

c) el crecimiento de la población activa en el período.

i) y ii) del punto $b$ ) pueden tomarse conjuntamente de acuerdo con la interpretación y significado de los datos.

Asimismo, la demanda de mano de obra en el análisis por incrementos es conceptualmente más complicada, en especial en el análisis de las inversiones, sin embargo es muy interesante desde el punto de vista académico. Se presentan y discuten ambos modelos, pero dado que cierta información sobre la demanda de mano de obra en el análisis de incrementos, específicamente la matriz $B$, no es fácilmente disponible, en el modelo de incrementos se especificará empíricamente solamente la parte de la oferta. Esto tiene su propio valor ya que proporciona información adicional la cual no se especifica en el análisis en términos absolutos. El análisis de incrementos se denomina modelo $1 \mathrm{y}$ el análisis en términos absolutos modelo 2 . El primero se basa sustancialmente en un modelo desarrollado por Sandee para la economía de la India de 1960 [8].

Modelo 1. El principal objetivo de este modelo es estimar la demanda de mano de obra para 1982. El modelo trabaja a base de incrementos entre 1970 y 1982 para obtener la demanda final. A través de este incremento en la demanda final se determina la producción bruta correspondiente y de aquí se deriva el correspondiente valor agregado. Al estimar los coeficientes agregados para 1982, y multiplicados por el incremento en el valor agregado por sector, se obtiene el incremento correspondiente al empleo de 1970 a 1982.

En seguida se calcula el incremento en la oferta de trabajo en ese mismo período, y al comparar los incrementos en la oferta y la demanda de trabajo hasta 1982 se puede estimar el desempleo.

Dado que éste es el propósito general del modelo, se hace ahora necesario discutir algunos aspectos con mayor detalle. 
De acuerdo con la ecuación 1 del Apéndice, la demanda final incluye el consumo tanto privado como gubernamental, las exportaciones menos las importaciones, y las inversiones. Como se mencionó, el modelo trabaja con el incremento en la demanda final al comparar 1970 con 1982. Las relaciones con la inversión son algo más complejas, ya que el incremento en la inversión depende del incremento de otros componentes de la demanda final hasta 1982.

La ecuación 2 especifica que el incremento tatal en las inversiones $(K)$ es igual a la matriz de inversiones totales $(B)$, multiplicada por el incremento en la producción bruta, esto es:

$$
K=B \Delta X
$$

donde:

$$
B \Delta X=T\left(K_{o}+\frac{1}{2} \Delta K\right)
$$

La gráfica 1 puede auxiliar a visualizar cómo se formulan los incrementos en las inversiones de 1970 a 1982.

\section{Gráfica 1}

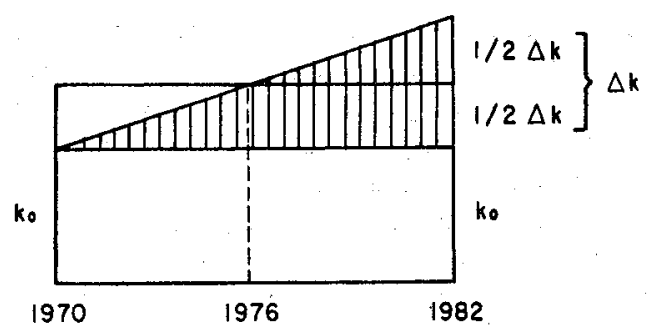

$K_{o}$ es la inversión necesaria para lograr la producción de 1970. La necesidad de este nivel de inversión permanece constante en cada año hasta 1982, siendo entonces el total necesitado de $12 K_{o}$.

El incremento en la inversión del período 1970-1982 se indica por el triángulo sombreado, el cual corresponde a un incremento en la producción final sobre el mismo período. Esta cantidad es aproximadamente:

$12(1 / 2 \Delta K)$ o sea $6 \Delta K$

por lo que $K$ es en este caso:

$$
K=12 K_{o}+12(1 / 2 \Delta K)
$$

o en términos más generales:

$$
K=T K_{o}+\frac{1}{2} T(\Delta K)
$$

esto es: 


$$
\begin{aligned}
& K=T\left(K_{o}+\frac{1}{2} \Delta K\right)=B \Delta X \\
& 2 B \Delta X=2 T\left(2 K_{o}+\Delta K\right) \\
& \frac{2 \mathrm{~B} \Delta X}{T}=2 K_{o}+\Delta K \\
& \frac{2 B \Delta X}{T}=2 K_{o}=\Delta K
\end{aligned}
$$

Esta ecuación se denota como (2.1) en el apéndice.

La matriz $B$ necesaria para el cálculo es aquella que se expresa en términos absolutos y no aquella expresada en incrementos o tasas de crecimiento, y no debe incluir sólo el capital fijo sino también las necesidades de reemplazo de capital y el capital activo. Ya que no se dispone de esta matriz, la demanda de mano de obra mediante el modelo 1 no puede estimarse.

Conceptualmente este modelo presenta cierta complejidad proveniente del hecho de que estamos estimando el incremento en la oferta y demanda de trabajo en un año futuro. Estimar para un año futuro la oferta y demanda de trabajo totales es un procedimiento más directo, y lo mejor es efectuar ambas estimaciones de modo de poder confrontar ambos resultados. Por desgracia esto último no se pudo lograr para el presente análisis. En el apéndice de este artículo se presentan los modelos, en el primero se hace el análisis por incrementos, y en el segundo el de la oferta y demanda de mano de obra totales para 1982.

La oferta de mano de obra en 1982 (ecuación 6), del análisis por incrementos; está constituida por varios elementos, el más importante de los cuales es el incremento de la PEA entre 1970 y 1982, es decir $\Delta \mathrm{N}$ (ecuación 6.2). Sin embargo, un aspecto sobre el nivel de empleo utilizado en la producción de 1970, y el cual está implícito en el análisis en términos absolutos, y que debe tomarse en cuenta explícitamente en el análisis por incrementos, es el cambio en la productividad de la mano de obra en el intervalo 1970-1982 (ecuación 6.1).

Un tercer elemento de la oferta de mano de obra es el número de los desempleados totalmente en 1970. En contraste con los otros dos factores de la oferta, éste no es un valor de incremento, aunque es evidente que debe formar parte de la oferta de trabajo para determinar el desempleo total en 1982. Esta cantidad figura en la ecuación 6 bajo la notación $\mathrm{LU}_{\text {io }}$

\section{Resultados empíricos del modelo 1}

El Censo General de Población de 1970 presenta el desglose de la PEA al 10. de enero de 1970. Dado que se utiliza para el análisis una matriz de insumo-producto de $15 \times 15$, sólo se toman las sumas del empleo por segmentos: 
PEA 1970

Gobierno no especificado

empleo sectorial
12955056

406607

747525

Se estima un empleo sectorial de 11.8 millones. Sin embargo en esta cifra se ocultan altos niveles de desempleo y de subempleo. El censo de 1970 consigna que de la PEA total, 485000 gentes estaban totalmente desempleadas. En un estudio reciente [9] con base en el censo de 1970, se estimaba en 5.8 millones el número de subempleados en 1969. Ese mismo estudio estima el equivalente del subempleo en términos de desempleo que resulta de 3 millones, como consecuencia de un desempleo total de 3485000 al 10 . de enero de 1970 .

Se supone que los datos del censo de 1970 sobre empleo sectorial incluyen subempleo, suposición cierta sin duda alguna, lo cual implica que los coeficientes del valor agregado de la mano de obra por sector también incluyen subempleo. Aún más, suponemos que los totalmente desempleados no están incluidos en los datos de empleo sectorial, y hay razones para suponer que en gran parte se incluye en el grupo de "no especificado". A esto se añade que en el sistema mexicano de insumoproducto no se incluyen las actividades puramente gubernamentales, y entonces tampoco se incluye en este análisis el empleo correspondiente.

De esta manera, la parte de la PEA considerada en el análisis es, al 10. de enero de 1970:

Empleados sectoriales
Desempleados
Total$$
\begin{array}{r}
11800924 \\
485000 \\
\hline 12 \quad 285924
\end{array}
$$

El crecimiento de la PEA en el período bajo consideración se estima en $3.8 \%$ al año. En consecuencia, la parte de la PEA considerada en enero 1o. de 1982 es:

$\begin{array}{ll}1982 & 19220993 \\ 1970 & 12285924 \\ \Delta \mathrm{N} & 12\end{array}$

Esta cantidad, redondeada en 6935000 figura en las ecuaciones 6 y 6.2 con el símbolo $\Delta \mathrm{N}$.

Como se mencionó, los 485000 desempleados en 1970 entran dentro del cálculo de la oferta de mano de obra para 1982 para determinar el número total de gastos para los cuales el sistema económico debe proveer trabajo.

Por último, debe determinarse la fuerza de trabajo de la total existente en 1970 que se hace innecesaria debido al aumento de la productividad en 1982. La ecuación 6.1 especifica el procedimiento para determinar el número de trabajadores sustraídos. Los coeficientes de valor agregado de la mano de obra sectorial para 1982 deben sustraerse de 
los de 1970 y la diferencia resultante se multiplica por el valor agregado de 1970.

La proyección de coeficientes de mano de obra se llevó a cabo como sigue: se disponía de datos de empleo sectorial en 1969 y una proyección para 1976, así como de datos de los valores agregados correspondientes. La información pertinente se presenta en los cuadros 1 y 2 .

\section{Cuadro 1}

MÉxico: VAlor agregado BRUTo (Millones de pesos a precios de 1960)

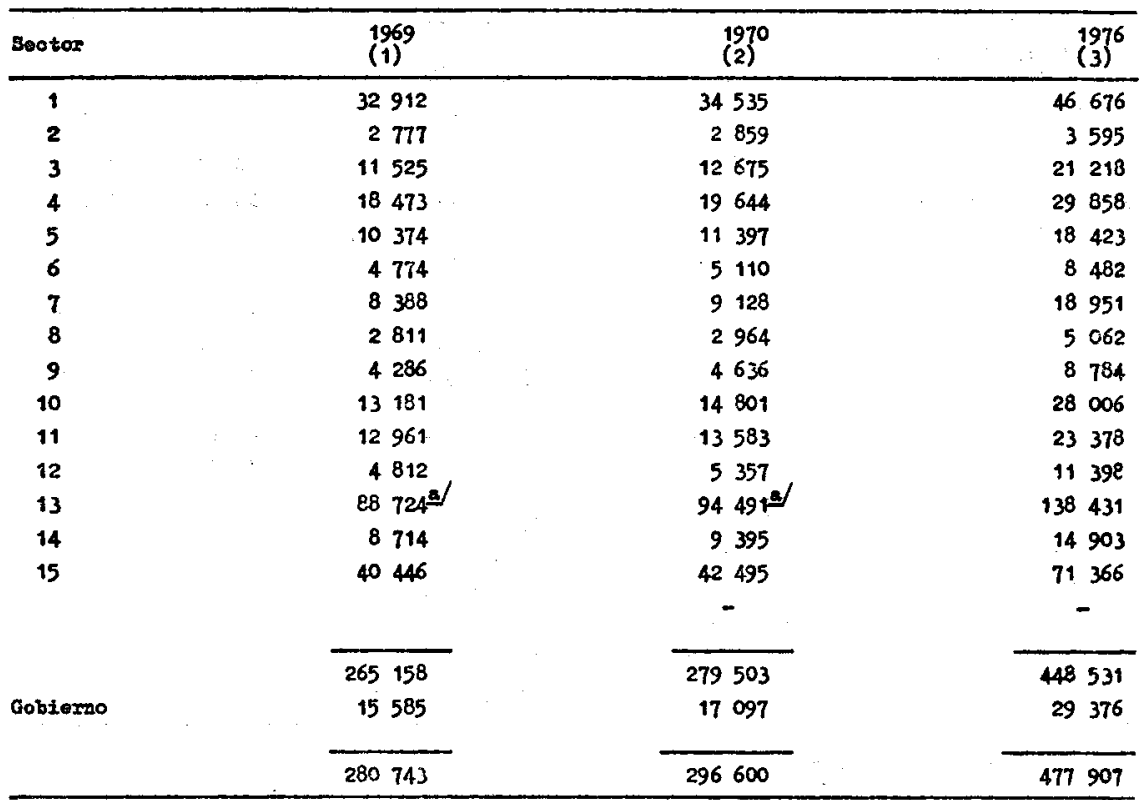

Fuentes (1): Banco de México, Informe Anual, 1970.

(2) y (3): véase Ref. [9]

a Sólo se incluye comercio al mayoreo y menudeo, restaurantes y hoteles, en el sector de servicios (15).

De estos datos se obtuvieron los coeficientes de valor agregado de la mano de obra, y se determinaron para cada sector las tasas de crecimiento (negativas) para el período 1969-1976. Por medio de estas tasas de crecimiento se interpolaron y extrapolaron los coeficientes del valor agregado de la mano de obra para el lapso 1970-1982 - los resultados se presentan en el cuadro 2. Desde luego que la confiabilidad de los datos debe tomarse con reservas. Los coeficientes así proyectados los denotamos como I.

Sería también interesante proyectar los coeficientes de mano de obra de modo totalmente independiente para evitar toda la endogamia de una proyección de datos a nivel nacional. Esta posibilidad existe gracias 
Cuadro 2

MÉxico: NúMERo de trabajadores Y COEFicientes de MANo DE OBRA PROYECTADOS

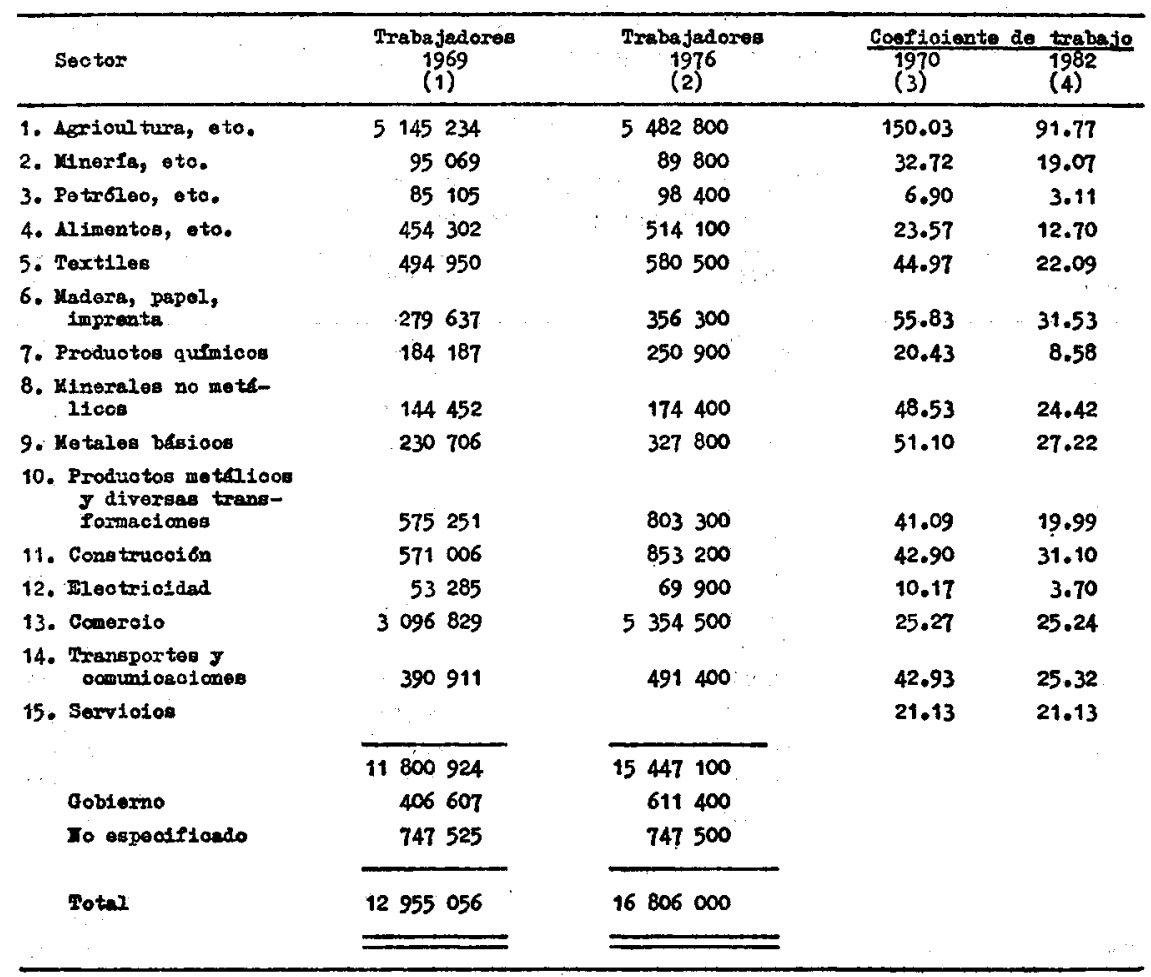

Fuente: Columnas 1 y 2: Ref. [9].

Columnas 3 y 4: Información proyectada presentada en el cuadro 1 y Ref. [5].

a las funciones de regresión desarrolladas en [5] para los 7 sectores de transformación así como para minería y construcción. Los coeficientes de trabajo, tipo II, se proyectaron en esta forma y se presentan en el cuadro 4. Para los sectores restantes, para los que no tenemos funciones de regresión, se utilizan los coeficientes de empleo (I).

Como ya se mencionó, la ecuación 6.1 sirve para estimar la fuerza de trabajo que se libera de la existente en 1970, debido al incremento en la productividad del trabajo a lo largo del tiempo, y esto supone un incremento de la productividad de la mano de obra en cada sector. En principio, los coeficientes de trabajo podrían moverse en dos direcciones: hacia un mayor uso de la mano de obra, o hacia uno menor. Asimismo, el cambio en los coeficientes de trabajo no tienen que ser en la misma dirección para cada sector.

Sin embargo, lo más factible es que se mantenga la tendencia actual, 
y que los coeficientes de mano de obra se reduzcan paulatinamente para el mismo nivel de producción, implicando mayor productividad de la mano de obra a través del tiempo. Por ello, se adopta esta posición como punto de partida en esta parte del análisis. Este punto de arranque parece bastante realista, y más aún si se considera que tales incrementos en la productividad de la mano de obra no necesariamente son producto de un mayor capital de producción, sino que puede ser resultado sólo de la madurez de la mano de obra, tanto en sentido vocacional como profesional. Por estas razones, la fuerza de trabajo necesaria para la producción del año base 1970 se reducirá gradualmente. Sin embargo, se hace necesario prestar más atención a los sectores de comercio y servicios, 13 y 15 respectivamente.

Como se dijo, se dispuso de información básica sobre el valor agregado y el nivel de empleo para los años 1969 y 1976 [9]. Sin embargo, sólo se disponía de datos sobre el nivel de empleo en los sectores de comercio y servicios en forma conjunta. Tomando el valor agregado y el empleo para estos dos sectores juntos, el coeficiente de trabajo correspondiente mostró una tasa positiva de crecimiento medio anual de $7.25 \%$, en contraposición con todos los demás sectores que mostraron tasas de crecimiento negativas, y que traen como consecuencia coeficientes de trabajo menores para 1982. Si se obtienen los coeficientes de trabajo de 1969 mediante un método plausible tanto para el sector comercio como para el de servicios, y se utiliza para ambos la tasa de crecimiento del $7.25 \%$, los coeficientes de trabajo resultan como se aprecia en el cuadro 3.

Curadro 3

TRABAJADORES POR MILLÓN DE PESOS DE VALOR AGREGADO

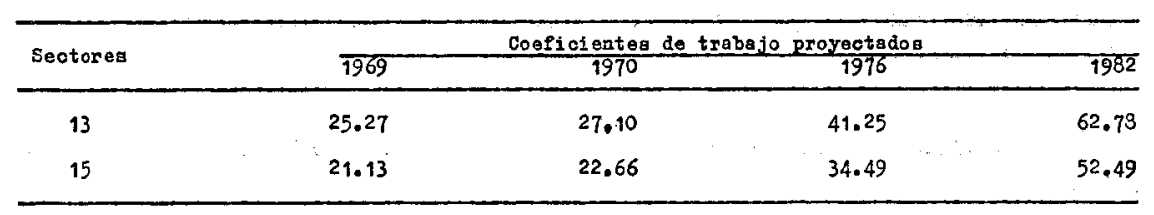

De acuerdo con la información básica utilizada, se concluye que una gran parte del desempleo y del subempleo es absorbido por actividades marginales de comercio y de servicios. No obstante, de acuerdo con criterios económicos, estos trabajadores son esencialmente subempleados. Por lo tanto, resulta inadecuado utilizar estos coeficientes sobrestimados de desempleo para estimar el desempleo en estos dos sectores en 1982. En su lugar se supone que el crecimiento de la productividad de la mano de obra en estos sectores será de cero en el período, y entonces se aplicarán para 1970-1982 los mismos coeficientes de mano de obra obtenidos para 1969. Incluso esto último presupone una cierta absorción intrínseca de mano de obra por parte de actividades marginales, lo cual en esencia implica subempleo. 
Si se multiplica la diferencia entre los coeficientes de trabajo sectoriales de 1970 y 1982 por el valor agregado en 1970 , el total de mano de obra sustraída de la existente en 1970, resulta de 3.7 millones, de acuerdo con los coeficientes (I), y de 3.5 millones de acuerdo con los coeficientes (II), tal y como puede deducirse del cuadro 4.

\section{Cuadro 4}

MÉxico: CoEficientes de tRABAjo tIPo II PROYEctados, Y FUERZA DE TRABAJO SUSTRAÍDA DE LA EXISTENTE EN 1970

\begin{tabular}{|c|c|c|c|}
\hline Seotox & $1982=$ & $\left.\underset{I}{\left(L_{70}-L_{82}\right.}\right)^{v_{70}}$ & ${ }_{I I}^{\left(L_{70}-L_{82}\right)^{\nabla} 70}$ \\
\hline b & - & 2012009 & 2012009 \\
\hline 2 & 16.52 & 39025 & 46396 \\
\hline $3^{b}$ & - & $\begin{array}{lll}48 & 038\end{array}$ & $\begin{array}{ll}48 & 038\end{array}$ \\
\hline 4 & 20.16 & $20960 t$ & 63057 \\
\hline 5 & 39.71 & 260763 & $151 \quad 124$ \\
\hline 6 & 23.92 & 124.173 & 163060 \\
\hline 7 & 12.87 & $108 \quad 167$ & 69008 \\
\hline 8 & 21.07 & 71462 & 81391 \\
\hline 9 & 16.38 & 110708 & 160967 \\
\hline 10 & 23.57 & 312301 & 259314 \\
\hline 11 & 25.94 & $160 \quad 144$ & 34660 \\
\hline $12^{\mathrm{y} /}$ & - & 34660 & - \\
\hline $13^{b /}$ & - & - & 165446 \\
\hline $14^{b}$ & - & 165446 & - \\
\hline $19^{b /}$ & - & - & \\
\hline Total & & 3656497 & 3484753 \\
\hline
\end{tabular}

a Proyectado mediante funciones de regresión; véase [5].

b Para los sectores 1,3 y 12 a 15 , se utilizan los mismos coeficientes de trabajo que en el cuadro 2 . sigue:

El incremento total de la oferta de trabajo en 1982 se estima como

Oferta de trabajo $\left(L_{s}\right)_{82}$

i) incremento de la PEA

6935000

ii) desempleo en 1970

485000

iii) sustracción de fuerza de trabajo de la de 1970 (promedio de ambas estimaciones)

Total

Como se señaló, no vamos a especificar empíricamente la demanda de mano de obra con el modelo 1. Cualquiera que sea la demanda $-\mathrm{y}$ en esencia el desempleo implícito, que resulte de deducir el incremento en la demanda de mano de obra del incremento en la oferta, y que debe coincidir con el desempleo estimado con el modelo 2- debe hacerse una corrección con base en el nivel de empleo que se encuentre en los 
sectores comercio y servicios. Cmo ya se mencionó, si se mantienen constantes los coeficientes de mano de obra de 1970 a 1982 , el subempleo se manifestará esencialmente en estos sectores y no debe considerarse como empleo.

El empleo total para 1982 se estima en estos dos sectores como se observa en el cuadro 5 .

Cuadro 5

\begin{tabular}{cc}
\hline Sector & Millones de trabejadores \\
\hline 13 & 2.25 \\
15 & 1.74 \\
Total & 3.99 \\
\hline
\end{tabular}

Puede suponerse con toda seguridad que alrededor de un tercio de éstos estarán subempleados. Al suponer un subempleo del $70 \%$ del tiempo, se añaden 900000 más a los totalmente desempleados.

\section{Resultados empíricos del modelo 2}

En el modelo 2 se compara la oferta y demanda de mano de obra en 1982. Mediante una proyección de la demanda final para 1982, se obtiene el correspondiente producto bruto de 1982 al multiplicar la demanda final por la inversa de $(\mathrm{I}-\mathrm{A}-\mathrm{B})$. De nuevo, se debe tomar en cuenta la parte de las inversiones para la demanda final. Se desea estimar la inversión en 1982 en lugar del total de inversiones. Utilizando una idea de Sandee [4] la que se aplicó en un estudio del Banco de México [2], [3], la inversión en 1982 puede estimarse si se multiplica la matriz $B$ por las tasas estimadas de crecimiento anual de la producción bruta por sectores en 1982: $\delta x$, en consecuencia:

$$
(\vec{B})=(B) \delta x
$$

Al utilizar la matriz $(\vec{B})$ en lugar de la $(B)$ se están utilizando las inversiones en 1982 en lugar del total de las inversiones. En el cuadro 6 se presentan los niveles de empleo proyectados por sectores y de acuerdo con la aplicación de los dos coeficientes de mano de obra. El total de trabajadores no presenta gran diferencia con cada uno de esos coeficientes de mano de obra utilizados, lo cual se debe en parte al hecho de que para los sectores agrícola, comercial y de servicios, que utilizan intensivamente la mano de obra, se aplican los mismos coeficientes. Para minería y construcción las estimaciones arrojan resultados parecidos. Por otra parte, entre las industrias de transformación existen diferencias sustanciales: como son por ejemplo los sectores 4,5 , y 10 que respectivamente corresponden a las industrias alimencia, de textiles, y de productos me- 
tálicos. Sin embargo, no hay una tendencia clara ya que para ciertos sectores los coeficientes de mano de obra del tipo $I$ estiman mayor nivel de empleo, y para otros sectores es el tipo II de coeficientes el que da las mayores estimaciones. En ambos casos el total estimado es semejante y podemos inferir que el nivel total de empleo es más confiable que el desgloce.

\section{Cuadro 6}

México: Estimación del NiVEL de eMpleo en 1982 (Modelo 2) (miles de trabajadores)

\begin{tabular}{crr}
\hline Sector & I & II \\
\hline 1 & 6836 & 6836 \\
2 & 121 & 104 \\
3 & 112 & 112 \\
4 & 486 & 772 \\
5 & 464 & 667 \\
6 & 469 & 356 \\
7 & 210 & 315 \\
8 & 131 & 113 \\
9 & 262 & 158 \\
10 & 794 & 936 \\
11 & 770 & 642 \\
12 & 68 & 68 \\
13 & 4916 & 416 \\
14 & 619 & 618 \\
15 & 2764 & 2764 \\
Total & 19020 & 19376 \\
\hline
\end{tabular}

De acuerdo con este modelo la demanda total de mano de obra en 1982 será de:

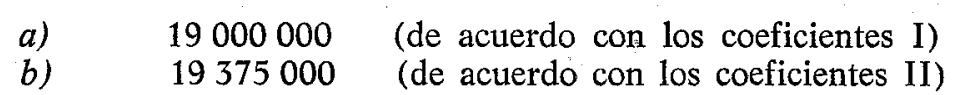

Dado que la PEA de 1982, ha sido estimada para este análisis en 19221000 , se puede concluir que la oferta y la demanda se encontrarán prácticamente en equilibrio, y que el único desempleo es el que quedaría confundido en los sectores 13 y 15 y que estimamos en alrededor de un millón.

De acuerdo con estos resultados, el desempleo será de apenas 5\% de la población activa, 27\% del que había en 1970.

Sin embargo, este resultado presupone una tasa de crecimiento global del PIB, y ésta pudiera ser muy optimista. A partir del siguiente punto, que se ocupa de la capacidad de absorción de mano de obra de la economía mexicana en el año 2000 , puede verse que el nivel de empleo es muy sensible a las tasas de crecimiento tanto globales como sectoriales. 


\section{LA CAPACIDAD DE ABSORCIÓN DE MANO DE OBRA EN EL AÑo 2000}

Es interesante extender el estudio de este fenómeno en México y mirar más al futuro, por ejemplo hasta el año 2000. Esto puede hacerse aunque de modo más aproximativo, pero en lugar de trabajar con un desglose de 15 sectores, se usará una composición sectorial más agregada, para cuyo análisis se distinguen seis sectores que se definen como sigue:

\section{Agricultura y minería}

II Transformación

III Energéticos: petróleo, carbón y electricidad

IV Construcción

V Transportes

VI Comercio y servicios, excluidos los del gobierno.

Para estos seis sectores agregados se determina el valor agregado y el nivel de empleo para los años 1960,1969 y 1976. Se excluyen tanto el valor agregado como el nivel de empleo de las actividades gubernamentales. Se incluye el sector público productivo -y se excluyen las actividades administrativas del gobierno como son las secretarías de estado. De igual manera se excluye del nivel de empleo el grupo censal marcado como "no especificado".

Para cada uno de los seis sectores se determinan las tasas de crecimiento para el valor agregado y para los coeficientes del valor agregado de la mano de obra. Tales tasas de crecimiento aparecen en los cuadros 7 y 8 .

\section{Cuadro 7}

MÉxico: TASAS DE CRECIMIENTO del VALOR AGREgado

\begin{tabular}{|c|c|c|c|c|c|}
\hline & Seotor & $1960-1969$ & $1969-1976$ & $1960-1976$ & $1976-2000$ \\
\hline $\begin{array}{l}\text { I } \\
\text { II } \\
\text { III } \\
\text { IV } \\
\text { VI } \\
\text { VII }\end{array}$ & 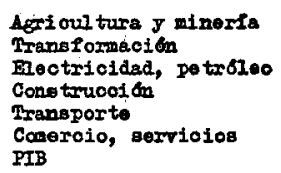 & $\begin{array}{r}3.46 \\
8.91 \\
10.53 \\
8.72 \\
6.38 \\
6.38 \\
6.75\end{array}$ & $\begin{array}{r}5.02 \\
9.50 \\
10.39 \\
8.79 \\
7.97 \\
7.17 \\
7.80\end{array}$ & $\begin{array}{r}4.14 \\
9.17 \\
10.47 \\
8.75 \\
7.07 \\
6.94 \\
7.33\end{array}$ & $\begin{array}{r}4.72 \\
9.39 \\
10.42 \\
8.78 \\
7.67 \\
7.09 \\
7.64\end{array}$ \\
\hline
\end{tabular}

Las tasas de crecimiento para el período 1976-2000 se obtienen de tasas de crecimiento previas mediante procedimientos de ponderación, asignando mayor peso a las tasas esperadas de crecimiento y menor a las tasas de crecimiento pasadas.

Las tasas de crecimiento de la productividad de la mano de obra y las tasas de crecimiento de los coeficientes de valor agregado de la 
Cuadro 8

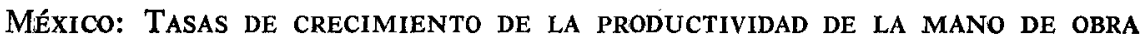

\begin{tabular}{cccccc}
\hline Seotor & $1960-1969$ & $1969-1976$ & $1960-1970$ & $1976-2000$ & $\begin{array}{c}\text { Coeficiente } \\
\text { mano da obra/ralor agre } \\
\text { 8ado proyectado }\end{array}$ \\
\hline II & 4.05 & 4.09 & 4.07 & 4.15 & 41.80 \\
III & 3.90 & 5.77 & 4.07 & 5.21 & 7.58 \\
IV & 7.06 & 7.27 & 7.15 & 7.23 & .97 \\
VI & 4.02 & 2.74 & 3.45 & 2.98 & 18.04 \\
VI & 4.99 & 4.54 & 4.80 & 4.63 & 11.10 \\
\hline
\end{tabular}

mano de obra son idénticos excepto por el signo negativo ya que estos últimos coeficientes son el inverso de las primeras tasas.

En la última columna del cuadro 8 se presentan los coeficientes de valor agregado de la mano de obra, y se indica el número de trabajadores necesarios para producir un millón de pesos de valor agregado a precios de 1960. Se pueden observar grandes diferencias en las tasas: por ejemplo, se requiere menos de un hombre para producir un millón de pesos de valor agregado en los sectores de energéticos en el año 2000 , mientras que para producir un millón de pesos de valor agregado de productos primarios se requieren alrededor de 42 trabajadores.

\section{Absorción de empleo para varias tasas de crecimiento del PIB}

En el cuadro 9 se proyectan al año 2000 el valor agregado (a precios de 1960) y el empleo a partir de 1976 con las tasas de crecimiento especificadas en los cuadros 7 y 8 .

\section{Cuadro 9}

México: PIB Y EMPLEO EN EL AÑo 2000 (I)

\begin{tabular}{|c|c|c|c|c|}
\hline & \multicolumn{2}{|c|}{ Valor agregado bruto } & \multirow{2}{*}{ 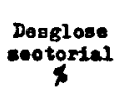 } & \multirow{2}{*}{$\begin{array}{c}\text { Bmploo } \\
\text { (ailea de trabajadorea) } \\
2000\end{array}$} \\
\hline & 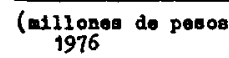 & de $\begin{array}{c}1960) \\
2000\end{array}$ & & \\
\hline $\begin{array}{c}\text { I } \\
\text { II } \\
\text { III } \\
\text { IV } \\
\text { VI } \\
\text { PI } \\
\text { PIB }\end{array}$ & $\begin{array}{rr}50 & 271 \\
117 & 566 \\
32 & 616 \\
23 & 378 \\
14 & 903 \\
209 & 797 \\
448 & 531\end{array}$ & $\begin{array}{rrr}152 & 075 \\
1 & 013 & 337 \\
352 & 031 \\
176 & 198 \\
87 & 816 \\
87 & 816 & 888 \\
1 & 085 & 888 \\
2 & 867 & 345\end{array}$ & $\begin{array}{r}5.3 \\
35.3 \\
12.3 \\
6.1 \\
3.1 \\
37.9 \\
100.0\end{array}$ & $\begin{array}{rr}6 & 357 \\
7 & 681 \\
& 341 \\
3 & 161 \\
& 975 \\
21 & 750 \\
40 & 265\end{array}$ \\
\hline
\end{tabular}

A partir de esta proyección resulta que el empleo en México en el año 2000 sería de alrededor de 40 millones de personas si el valor agregado y la productividad del trabajo crecen como se indica en los cuadros 6 y 7 . Deberá tomarse en cuenta que estas tasas de crecimiento se derivan de tendencias pasadas y por lo tanto no son del todo irreales. Por su parte, el valor agregado bruto a precios constantes se incrementa de 448.5 y 2867.3 miles de millones de pesos entre 1976 y el año 2000 , 
lo que implica una tasa de crecimiento global del PIB de alrededor de $8.04 \%$ anual.

Una vez determinada la demanda sectorial y total de empleo en el año 2000 , podemos analizar los aspectos de la oferta.

La PEA total en el año 2000 resulta de 39660 millones si se utiliza una tasa media de crecimiento anual de $3.8 \%$. Por lo tanto, la oferta y la demanda de empleo resulta balanceada. Sin embargo, como se recordará, se excluye al gobierno y a los empleos no especificados.

Por lo tanto, al utilizar la misma tasa de crecimiento del $3.8 \%$, resulta que la parte comparable de la PEA es de 37.8 millones, lo que sugiere que para el año 2000 México sufrirá un déficit de mano de obra de más o menos 2.5 millones, si se utilizan las tasas de crecimiento especificadas.

El desglose de la PEA, considerada en este análisis la empleada en el gobierno y la no especificada, se estiman para el año 2000 como sigue:

\begin{tabular}{lrc} 
PEA & 37.8 & millones \\
Empleados del gobierno & 1.3 & $"$ \\
No especificada & 0.6 & $"$ \\
\cline { 2 - 2 } & 39.7 & millones
\end{tabular}

Si se considera que los trabajadores en actividades no especificadas es de alrededor de 0.6 millones, el déficit de mano de obra será de más o menos 2 millones para el año 2000.

Por supuesto que no es posible adoptar políticas que tomen como base este pronóstico tan favorable, ya que todo tipo de acontecimientos pueden interferir con semejante prospecto de crecimiento tan elevado y sostenido. Más aún, no es totalmente predecible que la balanza de pagos y la capacidad de ahorro e inversión del país sean capaces de apoyar la alta tasa de crecimiento del $8.04 \%$ durante todo un cuarto de siglo.

Como consecuencia, para la segunda estimación (II) se modifican las tasas de crecimiento sectoriales, a excepción del primero, para obtener una tasa de crecimiento global del PIB de 7\% anual. En el cuadro 10 se presentan los resultados de tales cálculos.

Cuadro 10

México: PIB y Nivel de empleo en el aÑo 2000 (II)

\begin{tabular}{|c|c|c|c|c|}
\hline \multirow[b]{2}{*}{ Seotor } & \multicolumn{2}{|c|}{ Valor egregado bruto } & \multirow{2}{*}{$\begin{array}{l}\text { Desglooe } \\
\text { sootorial } \\
(x)\end{array}$} & \multirow{2}{*}{$\begin{array}{l}\text { Hivel de } \\
\text { empleo }\end{array}$} \\
\hline & $\begin{array}{l}\text { Tasa de } \\
\text { orvoimiento }\end{array}$ & $\begin{array}{l}\text { Millonos de posos } \\
\text { a prool os de } 1960\end{array}$ & & \\
\hline \multirow[t]{2}{*}{$\begin{array}{r}I I \\
I I I I \\
I V \\
V \\
V I\end{array}$} & $\begin{array}{l}4.72 \\
7.50 \\
7.50 \\
7.00 \\
7.00 \\
7.00\end{array}$ & $\begin{array}{r}152075 \\
666937 \\
185026 \\
118582 \\
75593 \\
1064 \quad 167\end{array}$ & $\begin{array}{r}6.7 \\
29.6 \\
8.2 \\
5.2 \\
3.3 \\
47.0\end{array}$ & $\begin{array}{rrr}6 & 357 & 000 \\
5 & 055 & 380 \\
179 & 480 \\
2 & 139 & 220 \\
& 839 & 080 \\
21 & 315 & 270\end{array}$ \\
\hline & $\sim 7.00$ & 2262380 & 100.0 & 35885730 \\
\hline
\end{tabular}


Una reducción en la tasa de crecimiento del PIB de 8.04 a $7.00 \%$ trae como consecuencia un desempleo de 2 millones, además de 0.6 millones de trabajadores con actividad no especificada, o sea, un total de 2.6 millones.

Una tasa de crecimiento de 7\% anual sostenida durante un cuarto de siglo es demasiado optimista como para que sea factible. Por tanto, se examina una tercera alternativa en la que se utiliza una tasa de crecimiento anual del $6.4 \%$ cuyos resultados se presentan en el cuadro 11 .

Cuadro 11

MÉXICO: PIB Y NIVEL DE EMPLEO EN EL AÑo 2000 (III)

\begin{tabular}{|c|c|c|c|c|}
\hline \multirow{2}{*}{ Seotor } & \multicolumn{2}{|c|}{ Valor agregado bruto } & \multirow{2}{*}{$\begin{array}{l}\text { Desgloon } \\
\text {-otortal } \\
(\%)\end{array}$} & \multirow{2}{*}{$\begin{array}{l}\text { Nivel de } \\
\text { expleo }\end{array}$} \\
\hline & $\begin{array}{l}\text { Tasa de } \\
\text { orooimiento }\end{array}$ & $\begin{array}{l}\text { Millonos de posos } \\
\text { a prooion do } 1960\end{array}$ & & \\
\hline $\begin{array}{r}\text { II } \\
\text { III } \\
\text { IV } \\
\text { VI } \\
\text { VII }\end{array}$ & $\begin{array}{l}4.72 \\
7.50 \\
7.50 \\
6.0 \\
6.0 \\
6.0 \\
6.5\end{array}$ & $\begin{array}{r}152075 \\
666937 \\
185026 \\
94656 \\
60341 \\
6034 \\
849454 \\
2008489\end{array}$ & $\begin{array}{r}7.6 \\
33.2 \\
9.2 \\
4.7 \\
3.0 \\
42.3 \\
100.0\end{array}$ & $\begin{array}{rrr}6 & 357 & 000 \\
5 & 055 & 382 \\
179 & 475 \\
1 & 707 & 594 \\
& 669 & 785 \\
17 & 014 & 563 \\
30 & 983 & 799\end{array}$ \\
\hline
\end{tabular}

Este tercer cálculo muestra un desempleo de 6.8 millones además de los 0.6 millones de la categoría de no especificados, lo que aproximadamente arroja 7.5 millones de desempleados para tal época. Las estimaciones II y III muestan la extraordinaria sensibilidad que tiene el nivel de empleo ante fluctuaciones en la tasa global de crecimiento del PIB. Esta sensibilidad se puede apreciar en las cifras del cuadro 12.

Cuadro 12

MÉxico: EMPLeo y DESEMPleo TOTAles EN FUNCión de la tASA DE CRECIMIENTO DEL PIB

\begin{tabular}{|c|c|c|c|}
\hline \multirow{2}{*}{$\begin{array}{l}\text { Tasa media anual do } \\
\text { orecimiento dol PIB } \\
\text { 1976-2000 }\end{array}$} & \multicolumn{3}{|c|}{ Año 2000} \\
\hline & \multicolumn{2}{|c|}{ Fapleados $_{\text {(millarea) }}^{\text {Desemplead os }}$} & \multirow{2}{*}{$\begin{array}{l}\begin{array}{l}\text { Deficit de } \\
\text { mano de obra }\end{array} \\
2000000 \\
-\end{array}$} \\
\hline $\begin{array}{l}8.04 \\
6.97 \\
6.45\end{array}$ & $\begin{array}{ll}40 & 265 \\
35 & 886 \\
30 & 984\end{array}$ & $\begin{array}{ll}\sim 2 & \overline{600} \\
\sim 7 & 500\end{array}$ & \\
\hline
\end{tabular}

a Se incluye la categoría de "no especificados".

Una diferencia tan pequeña en la tasa global de crecimiento del PIB, como la existente entre 6.45 y 8.04 , provoca que la economía sufra un vuelco de una situación con un fuerte déficit de mano de obra a una posición de desempleo agudo, con una diferencia total cercana a 10 millones de empleos anuales. En la gráfica 2, construida con base en el cuadro 12 , se presenta esta situación. 


\section{GráFICA 2}

MÉxico: Oferta Y DEMANDA DE EMPLEO, AÑo 2000

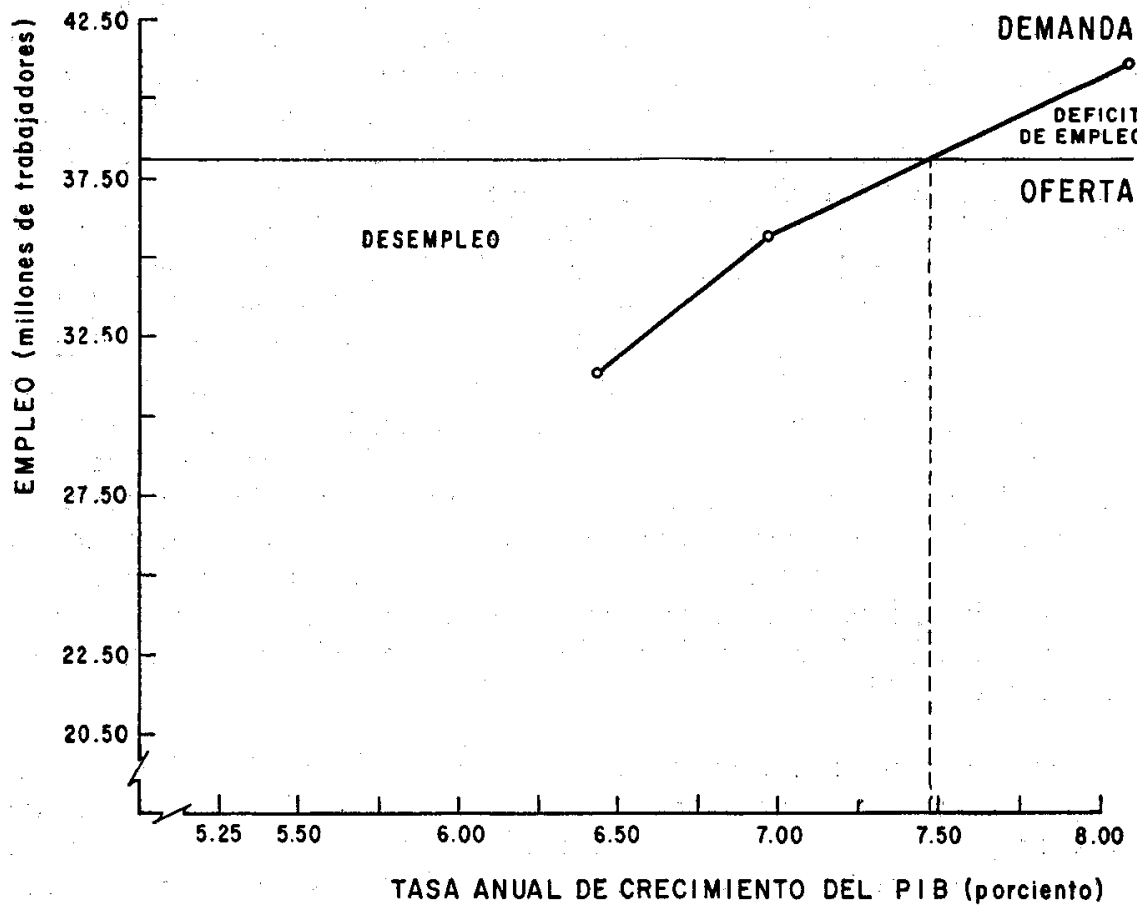

Una tasa de crecimiento anual media de $7.5 \%$ igualaría la oferta y la demanda de mano de obra si se considera la productividad que se especifica en el cuadro 8. Con todos los factores constantes, una tasa de crecimiento del PIB reducida implica desempleo, y una elevada trae como consecuencia déficit de mano de obra. Sin embargo, en cualquiera de las dos situaciones puede haber correcciones. Los sectores intensivos en el uso de mano de obra todavía tienen productividad bastante baja, y en el caso de déficit, una mayor productividad en estos sectores puede proveer fácilmente la necesaria para subsanar este déficit.

Ya que para el año 2000 es más factible una situación de desempleo que una de déficit de mano de obra, se pondrá mayor atención al problema de cómo corregir esta situación.

\section{Alternativas para asegurar empleo pleno}

Esta situación de desempleo puede corregirse mediante las siguientes políticas, o alguna combinación de éstas. 
Tipo de tecnología. Como puede verse en el cuadro 8 , se proyectaron al año 2000 incrementos anuales de la productividad bastante significativos en los seis sectores considerados. Estos importantes incrementos anuales en la productividad no pueden más que provenir, en su mayor parte, de fuertes inversiones en equipo productivo y no es seguro que el sistema económico pueda generar capital suficiente para sostener este elevado crecimiento en la productividad, especialmente al principio del período. Asimismo, al comenzar el período, la oferta de mano de obra sería más abundante y habría mayor desempleo. Por lo tanto, se aplicará más tecnología intensiva en mano de obra, lo cual implicará un menor crecimiento en la productividad de la mano de obra, mayor empleo, aunque también un menor crecimiento del pIB global, quizá entre el 6 y el $7 \%$.

Como se hizo notar, una tasa global de crecimiento del PIB del $6.45 \%$ puede provocar que 7.5 millones de la PEA queden desocupados, aunque esto presupone un crecimiento elevado de la productividad de la mano de obra con los correspondientes coeficientes de mano de obra más bajos. El crecimiento de la productividad del trabajo, la estructura de la producción y el crecimiento del PIB están interrelacionados de tal modo que si el PIB crece sólo $6.45 \%$ al año, entonces el crecimiento en la productividad de la mano de obra puede variar también, lo cual acarrea mayor ocupación. Por este simple hecho, puede reducirse el desempleo de manera considerable.

Estructura de la producción. La estructura de la producción nacional puede también cambiarse. Para el año 2000, los coeficientes de mano de obra proyectados, el inverso de la productividad de la mano de obra, varían de 42 trabajadores por cada millón de pesos de valor agregado en la agricultura a un trabajador en los sectores de generación de energía. Es claro entonces que un cambio ligero en las tasas relativas de crecimiento anual de los seis sectores afecten notablemente el nivel de ocupación. Una tasa de crecimiento algo mayor en los sectores intensivos en el uso de mano de obra como la agricultura, los servicios y la construcción con facilidad absorben dos millones de trabajadores.

Debe hacerse notar que al variar la tasa de crecimiento del PIB generalmente implica que también hay cambios en la estructura de la producción. Los tres cálculos presentados con tasas distintas de crecimiento del PIB, se ven afectados por cambios en las tasas relativas de crecimiento sectoriales, lo que trae consigo estructuras de la producción diferentes. No obstante, para una tasa de crecimiento del PIB y un tipo dado de tecnología, ambos fijos, también puede modificarse la estructura de la producción

Tasa de crecimiento de la PEA. Es obvio que el crecimiento de la PEA tendrá también una influencia decisiva en la oferta de trabajo en el año 2000. La tasa de crecimiento anual de la PEA, del 3.8\%, utilizada en estos cálculos, es extremadamente alta aunque realista para las condiciones actuales de México. Si para el año 2000 se pudiera reducir esta 
tasa al 3\% mediante una política efectiva de población a partir de 1975 , la parte de la PEA que se incluye en el análisis descendería en aproximadamente 7.5 millones. Si se supone que todos los demás factores permanecen constantes, prácticamente se eliminaría el desempleo en la tercera alternativa, esto es, un crecimiento del PIB del $6.45 \%$ combinado con altos niveles de productividad sectorial de la mano de obra.

Mediante' una combinación apropiada de los tres factores: tipo de tecnología, estructura de la producción y tasa de crecimiento de la población activa, puede eliminarse el desempleo para el año 2000. Esto también puede lograrse por el puro crecimiento del PIB para una estructura de la producción y un tipo de tecnología fijas. Sin embargo, no puede suponerse que no habrá interferencias económicas y políticas internas o externas que puedan amenazar una tasa de crecimiento elevada y sostenida del PIB, especialmente si la proyección cubre un cuarto del siglo. Por lo tanto parece más realista basar las futuras políticas económicas en un solo paquete organizado en el que todas las opciones discutidas jueguen su papel.

\section{APÉNDICE}

Modelo 1. Como punto inicial del análisis se tiene la relación básica en la cual el incremento en la producción total multiplicada por la matriz $(I-A)$ es igual al incremento en la demanda final, por lo que:

$$
(I-A) \Delta X=\Delta(C+E+K)
$$

donde: $\quad K=B \Delta X=T\left(K_{70}+1 / 2 \Delta K\right)$

y en consecuencia: $\Delta K=\frac{2 B \Delta X}{T}-2 K_{\mathrm{o}}$

$\Delta(C+E)$ se denota por $\Delta Y_{1}$, mientras que $E$ será $(E-M)$

por lo tanto la expresión 1) puede reescribirse como sigue:

$$
\begin{gathered}
\quad(I-A) \Delta X=\Delta Y_{1}+\frac{2 B \Delta X}{T}-2 K_{o} \\
\text { o: } \quad\left(I-A-\frac{2}{T} B\right) \Delta X=\left(\Delta Y_{1}-2 K_{o}\right) \\
\text { además: } \Delta X=\left(I-A-\frac{2}{T} B\right)^{-1}\left(\Delta Y_{1}-2 K_{o}\right)
\end{gathered}
$$

$(V / X) \Delta X=\Delta V$ 
En consecuencia, la demanda de mano de obra necesaria para generar este incremento en 1982 será:

$$
(L / V)_{82} \Delta V=\left(\Delta L_{d}\right)_{82}
$$

El incremento en la oferta de mano de obra en 1982 será:

$$
\begin{aligned}
& \quad L U_{70}+\Delta L_{70}+\Delta N=\left(\Delta L_{s}\right)_{82} \\
& \text { donde: }\left\{(L / V)_{70}-(L / V)_{82}\right\} V_{70}=\Delta\left(L_{70}\right) \\
& \text { y: } \quad N_{82}-N_{70}=\Delta N \\
& \quad \Delta L_{(70-82)} \leq \Delta L_{s}
\end{aligned}
$$

Modelo 2

$$
\begin{aligned}
& (I-A-\bar{B}) X=\dot{Y} \\
& \bar{B}=(B) \delta X \\
& Y_{82}=(C+E-M)_{82} \\
& X_{82}=(I-A-\bar{B})^{-1} Y_{82} \\
& (V / X) \cdot X_{82}=\dot{V}_{82} \\
& (L / V)_{82} . V_{82} \gtreqless N_{82}
\end{aligned}
$$

\section{BIBLIOGRAFIA}

[1] Banco de México, Departamento de Estudios Económicos, Cuentas nacionales y acervos de capital, 1950-1967, junio de 1969.

[2] Banco de México, Departamento de Estudios Económicos, Proyecciones de la economía mexicana para 1976, inédito, 1970.

[3] Banco de México, Departamento de Estudios Económicos, Proyecciones de los principales aspectos de la economía mexicana, inédito, 1970.

[4] Gerard K. Boon, Sector and Technology Choice in Economic Development, El Colegio de México, 1974.

[5] Gerard K. Boon, "Sustitución de capital y trabajo, comparaciones de productividad e insumos primarios y proyectados", Demografía Y Economía, Vol. VII, Núm. 3, 1973.

[6] Manne A. Goreux L., et al., Multi Level Planning: Case Studies in Mexico Amsterdam, North Holland Publishing, 1973.

[7] J. Sandee, "Ajuste del modelo sectorial", Banco de México, Departamento de Estudios Económicos, 1968.

[8] J. Sandee, A Demostration Planning Model for India, Nueva York, 1960.

[9] Grupo de Estudio del Problema del Empleo, El problema ocupacional en México, mimeografiado, 1973. 